\title{
Inspiration and Expiration Dynamics in Acute Emotional Stress Assessment
}

\author{
Javier Milagro ${ }^{1,2}$, Eduardo Gil ${ }^{1,2}$, Jorge M. Garzón-Rey ${ }^{2,3}$, Jordi Aguilón ${ }^{2,3}$, Raquel Bailón ${ }^{1,2}$ \\ ${ }^{1}$ BSICoS Group, Aragón Institute of Engineering Research (I3A), IIS Aragón, University of \\ Zaragoza, Spain \\ ${ }^{2}$ Centro de Investigación Biomédica en Red (CIBER), Madrid, Spain \\ ${ }^{3}$ Microelectronics and Electronic Systems Department, Autonomous University of Barcelona, \\ Bellaterra, Spain
}

\begin{abstract}
Relationships between inspiration and expiration times, $T_{i n}$ and $T_{e x}$, and inter-breath interval $(B B)$ were analyzed in a database composed by 120 young healthy volunteers undergoing a session divided in different stages aimed to induce relax and emotional stress, where respiration was recorded with a chest band. $T_{i n}$ and $T_{e x}$ were represented as a function of $B B$ so that two regression lines were obtained. The slopes of both regression lines, $\alpha_{T_{i n}}$ and $\alpha_{T_{e x}}$, as well as the angle formed by them, $\theta$, were used to characterize respiratory dynamics during each stage. Moreover, mean respiratory frequency in each stage was calculated. Statistical significant differences $(p<0.001)$ were obtained when comparing relax and stress stages, with increased $\alpha_{T_{e x}}$ and $\theta$, and reduced $\alpha_{T_{i n}}$ during stress. Also differences between distinct stress stages were found. Results suggest that inspiration and expiration patterns, and hence respiratory dynamics, are different under stress, so its analysis could be useful for stress assessment.
\end{abstract}

\section{Introduction}

Stress can be regarded as the complex physiological response of the body to a threatening situation. This general response, usually referred to as the "fight or flight" response, is mainly mediated by sympathetic nervous system and has its origin in the hypothalamus, which secretes a series of hormones that will modify the activity of the different systems composing the body. Changes in heart rate and heart rate variability (HRV) [1], blood pressure [2] or respiratory rate [3] among others have been assessed during stress. Although necessary, if this response to stress is either maintained in time or recurrent it could take the body away from its homeostatic state and hence lead to cardiovascular [4] or psychiatric disorders [5].

In the frame of the ES3 project [6], which aims to create a non-invasive tool for stress assessment, the focus of this work is in the respiratory system. In a previous study, respiratory rate analysis was proven to overcome HRV in stress assessment [3]. Our hypothesis is that not only respiratory rate, but also inspiration and expiration dynamics might be altered under stress. In this way, three indexes were proposed in order to characterize the dynamics of both processes in a database composed of volunteers undergoing stressful and non-stressful situations.

\section{Materials and Methods}

\subsection{Database}

A database of 120 healthy volunteers (60 male and 60 women) aged $21.99 \pm 2.87$ was acquired at University of Zaragoza, Autonomous University of Barcelona and Politechnic University of Madrid (40 subjects at each). Each subject underwent two different sessions, basal and stress, where respiratory signals were acquired with a chest band. Basal session consisted of a 10-minute baseline stage $\left(\mathrm{BL}_{\mathrm{B}}\right)$ followed by a 25 -minute relaxing stage $(\mathrm{RS})$, in both of which a relaxing audio was played. On the other hand, the stress session was divided in six stages. As subjects were expected to talk in two of them, thus producing noisy respiratory signals, only the following four stages were considered in the present study (further information about the whole protocol can be found elsewhere [3]):

1. Baseline stage $\left(\mathrm{BL}_{\mathrm{S}}\right)$ : 10-minute relaxing audio.

2. Story Telling (ST): three different stories with a big amount of details were told to each subject, who was requested to remember as many details as possible.

3. Stress Anticipation (SA): volunteers were kept waiting for the evaluation of the previous stage during 10 minutes.

4. Video Exposition (VE): subjects were shown a video with their performance in the memory test. Prior to the projection of the video, an actor perfectly repeating the stories was shown, so that subjects were made to believe that their performance was very poor. 
The former stage is considered as a basal state, whereas the rest of them are considered as stressful. Psychometric evaluation performed in [3] revealed that stress was indeed induced during stress session.

\subsection{Preprocessing}

First, respiratory signals, $x(t)$, were band-pass filtered between 0.03 and $0.8 \mathrm{~Hz}$ with a $3^{\text {rd }}$ order Butterworth filter, in order to eliminate the baseline and those highfrequency components that are not likely to be related with respiration. Forward-backward filtering was applied for preserving signal morphology. Afterwards, temporal events corresponding to the end of inspiration and expiration, $T_{P}$ (peak) and $T_{N}$ (nadir), were obtained from the filtered signals, $\hat{x}(t)$, as proposed in [7]. Essentially, two different moving average (MA) filters were applied to the filtered respiratory signal, thus resulting in two different signals:

$$
x_{W_{n}}(t)=\frac{1}{W_{n}} \sum_{j=0}^{W_{n}-1} \hat{x}(t-j)
$$

, where $W_{n}$ is the window length of each of the two MA filters and $n \in[1,2] . \quad W_{1}$ and $W_{2}$ were selected as 1.6 and 0.1 seconds respectively. In this way, $x_{W_{1}}(t)$ represents the slower trends of $\hat{x}(t)$, whereas $x_{W_{2}}(t)$ accounts for the faster trends. Those points where $x_{W_{2}}(t)$ crossed over $x_{W_{1}}(t)$ indicated an upward trend of $\hat{x}(t)$, whereas when $x_{W_{2}}(t)$ became lower than $x_{W_{1}}(t)$ a downward trend was found. Once these cross-points were located, $T_{P}^{j}$ was defined as the time occurrence of the maximum between an upward point and the following downward point for breath $j$, whereas $T_{N}^{j}$ was obtained as the time occurrence of the minimum between a downward point and the following upward point. In Fig. 1, the locations of the upward and downward trends of a given breath are shown.

\subsection{Respiratory dynamics}

Given a respiratory cycle, the total inspiratory amplitude, $A_{I}$, and the total expiratory amplitude, $A_{E}$, were defined over $\hat{x}(t)$ as the difference between the maximum volume of the inspiration and the minimum volume corresponding to the previous or the following expiration, respectively. Two time periods were defined for each respiratory cycle: $T_{i n}^{j}$ (inspiration) and $T_{e x}^{j}$ (expiration). $T_{i n}^{j}$ was obtained as the time that it takes to the respiratory signal to go from an amplitude of $0.1 A_{I}$ to $0.9 A_{I}$, whereas $T_{e x}^{j}$ was defined as the time that it takes to the respiratory signal to go from an amplitude of $0.9 A_{E}$ to $0.1 A_{E}$. These definitions of $T_{i n}^{j}$ and $T_{e x}^{j}$ were considered in order to reduce the variability that might be introduced in the calculation of $T_{P}$ and $T_{N}$. Therefore, $\mathbf{T}_{i n}$ was defined as

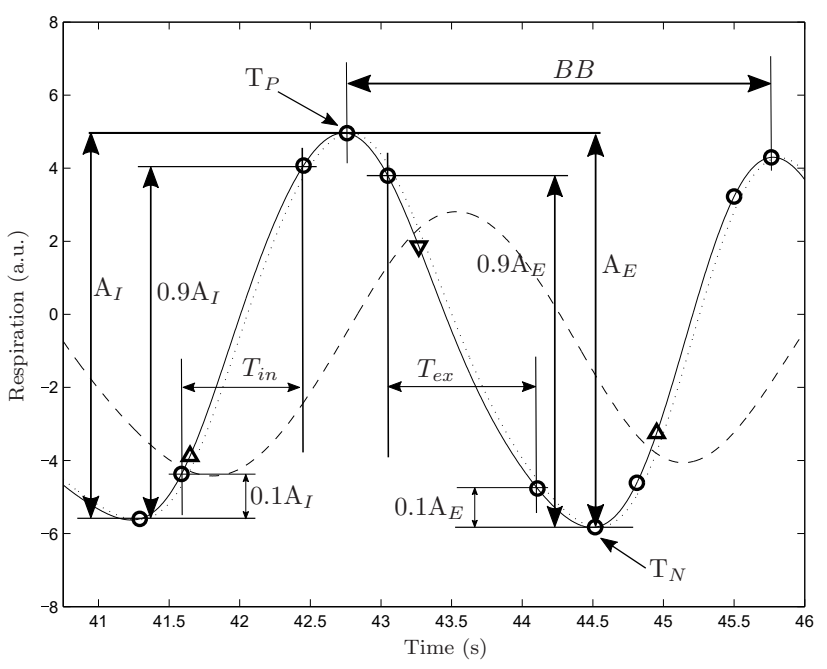

Figure 1. Example showing the definitions of $T_{i n}, T_{e x}$ and $B B$. The solid line represents $\hat{x}(t)$, whereas the dashed and dotted lines represent $x_{W_{1}}(t)$ and $x_{W_{2}}(t)$ respectively. The facing-up and facing-down triangles represent when an upward or downward trend have been found.

$\left[T_{i n}^{1} T_{i n}^{2} \cdots T_{i n}^{N}\right]$ and $\mathbf{T}_{e x}$ was defined as $\left[T_{e x}^{1} T_{e x}^{2} \cdots T_{e x}^{N}\right]$, being $\mathrm{N}$ the number of respiratory cycles of the recording. Also breath-to-breath (BB) interval series was defined as $\mathbf{B B}=\left[B B^{1} B B^{2} \cdots B B^{N-1}\right]$, being $B B^{j}=T_{P}^{j+1}-T_{P}^{j}$. An example showing the definition of these parameters is displayed in Fig. 1.

Afterwards, an outlier rejection algorithm was implemented in order to discard abnormal breaths from the analysis. Given one inspiration starting in $T_{N}^{j-1}$ and ending in $T_{P}^{j}$, and the subsequent expiration starting in $T_{P}^{j}$ and ending in $T_{N}^{j}, 3$ amplitude differences were computed: $A_{I}^{j}=T_{P}^{j}-T_{N}^{j-1}, A_{E}^{j}=T_{P}^{j}-T_{N}^{j}$ and $A_{N-N}^{j}=$ $\left|T_{N}^{j}-T_{N}^{j-1}\right| . \quad \mu_{A_{I}}$ and $\epsilon_{A_{I}}, \mu_{A_{E}}$ and $\epsilon_{A_{E}}$, and $\mu_{A_{N-N}}$ and $\epsilon_{A_{N-N}}$, were obtained as the mean and standard deviation of the previous 30 breaths for each of the magnitudes respectively. Running standard deviation outlier rejection was applied so that if any of the magnitudes laid out of the range $[\mu-\epsilon, \mu+\epsilon]$, breath $j$ was not considered in the analysis.

Respiratory system dynamics were characterized for each stage as the changes induced in $\mathbf{T}_{i n}$ and $\mathbf{T}_{e x}$ by a change in the BB interval, and they were quantified by representing both $\mathbf{T}_{i n}$ and $\mathbf{T}_{e x}$ as a function of BB. As the relationship between these magnitudes was found to be approximately linear, two lines were fitted: one for $\mathbf{T}_{i n}$ vs $\mathbf{B B}$ and another for $\mathbf{T}_{e x}$ vs $\mathbf{B B}$. The fitting was performed through the bisquare fitting method to increase robustness against outliers. Afterwards, the slopes of both lines, namely $\alpha_{T_{i n}}$ and $\alpha_{T_{e x}}$ respectively, were obtained. In this way, $\alpha_{T_{i n}}$ represents the variation that is expected 
to occur in the value of $T_{i n}^{j}$ given a change in the value of $B B_{j}$. Equivalently, $\alpha_{T_{e x}}$ represents the variation in $T_{e x}^{j}$ in response to a change in $B B_{j}$. Finally, the angle formed by the lines with slopes $\alpha_{T_{i n}}$ and $\alpha_{T_{e x}}, \theta$, was considered as a measurement of the difference between both slopes, being obtained as:

$$
\theta=\tan ^{-1}\left(\left|\frac{\alpha_{T_{i n}}-\alpha_{T_{e x}}}{1+\alpha_{T_{i n}} \alpha_{T_{e x}}}\right|\right)
$$

An example of $\alpha_{T_{i n}}, \alpha_{T_{e x}}$ and $\theta$ for the different stages of the stress session is shown in Fig. 2.

Additionally to mean $\mathrm{BB}$ interval $(\overline{B B})$, mean respiratory frequency, $\bar{f}_{\text {res }}$, was computed as in [3] so that results can be compared.

\subsection{Statistical methods}

Values of $\bar{f}_{r e s}, \overline{B B}, \alpha_{T_{i n}}, \alpha_{T_{e x}}$ and $\theta$ from the different stages of the protocol were compared. Hence, a Wilcoxon signed rank test was performed in order to assess if statistical significant differences could be found between stages. Statistical significance was considered for $p<0.001$. Also Spearman correlation between $\overline{B B}$ and the proposed parameters was obtained.

\section{Results}

Medians of each of the analyzed parameters obtained for $\mathrm{BL}_{\mathrm{B}}$ and $\mathrm{BL}_{\mathrm{S}}$ are displayed in Table 1. Statistical tests indicated that there were not significant differences in the considered parameters between the basal stage of both sessions. On the other hand, in Table 2 the values for each of the analyzed stages of the stress session $\left(\mathrm{BL}_{\mathrm{S}}, \mathrm{ST}, \mathrm{SA}\right.$ and VE) are shown. As can be noticed in Table 2, all the indexes were able to distinguish between $\mathrm{BL}_{\mathrm{S}}$ and all the stressing stages, with the exception of $\alpha_{T_{e x}}$ which showed no differences between $\mathrm{BL}_{\mathrm{S}}$ and $\mathrm{SA} . \overline{B B}$ was able to distinguish between ST, SA and VE, whereas $\bar{f}_{\text {res }}$ was able to distinguish between ST and VE and between SA and VE. Also $\alpha_{T_{e x}}$ and $\theta$ differed between ST and SA and between SA and VE. Finally, $\alpha_{T_{i n}}$ distinguished SA from VE. Median value of the different parameters was increased for ST, $\mathrm{SA}$ and VE when compared with $\mathrm{BL}_{\mathrm{S}}$ for all the parameters except $\alpha_{T_{i n}}$, which showed reduced median values for all the stressful stages. $T_{i n}$ and $T_{e x}$ also presented statistical significant differences between all the stages (except between ST and VE in the case of $T_{i n}$ ) with $p<0.001$, but they are not considered in the further discussion as they contain similar information than BB.

Spearman correlation between the proposed variables and $\overline{B B}$ is displayed in Table 3 , where absence of correlation can be noticed.

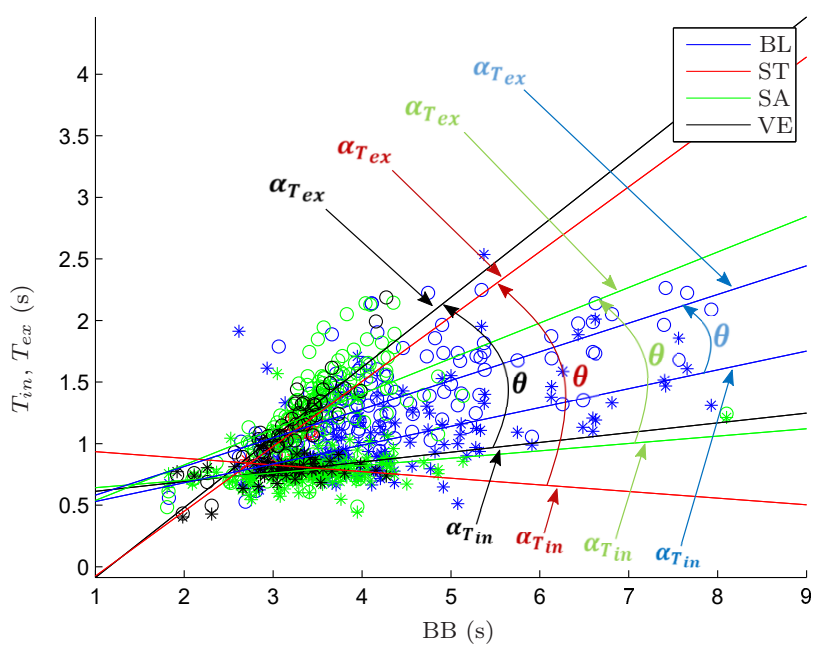

Figure 2. Example showing differences in $\alpha_{T_{i n}}, \alpha_{T_{e x}}$ and $\theta$ for the different stages of the stress session. Points corresponding to $T_{i n}$ are marked with asterisks, whereas points corresponding to $T_{e x}$ are labeled with empty circles.

\section{Discussion}

In a previous study, Hernando et al. employed HRV and respiratory rate analysis for assessing differences between the distinct stress stages considered here [3]. In [3], they found that respiratory rate overcame HRV analysis for this purpose, being able to discriminate between all the stages in the stress session. However, we hypothesized that autonomic control of respiratory system could change under stress conditions, and that this behavioral change does not only affect respiratory rate, but also respiratory dynamics.

As displayed in Table 1, the analyzed parameters were not significantly different between $\mathrm{BL}_{\mathrm{B}}$ and $\mathrm{BL}_{\mathrm{S}}$ stages, and so the use of $\mathrm{BL}_{\mathrm{S}}$ as a reference is justified.

The three proposed parameters are also shown in Table 2 to be able to distinguish between certain of the different stressful stages, being $\theta$ the one which distinguishes between more stages. This might reflect a dependency of the respiratory dynamics on the stress level, or in the attention level required in each stage (e.g., more attention is required in ST that in $\mathrm{SA}$ ). The fact that $\overline{B B}$ discriminates between more stages than $\bar{f}_{\text {res }}$ could be due to the highest intrinsic sampling frequency of $\mathrm{BB}$, which provides more observations than $\bar{f}_{\text {res }}$ : whereas one sample of $\mathrm{BB}$ is obtained for each breath, several breaths must be considered for estimating $\bar{f}_{\text {res }}$.

Results displayed in Table 2 suggest that respiratory dynamics are altered during stress. $\alpha_{T_{i n}}$ and $\alpha_{T_{e x}}$ are respectively thought to represent the adaptability of inspiration and expiration mechanisms when a change in BB takes place. On the other hand, $\theta$ would summarize the joint behavior of both processes. Consequently, changes in the 
Table 1. Median and IQR of the analyzed parameters during the baseline stages of the basal and stress sessions are shown. * reflects significant differences between stages with $p<0.001$.

\begin{tabular}{llllll} 
& $\bar{f}_{\text {res }}(H z) \overline{B B}(s)$ & $\alpha_{T_{i n}}$ & $\alpha_{T_{e x}}$ & $\theta(\mathrm{rad})$ \\
\hline $\mathbf{B L}_{\mathrm{B}}$ & 0.24 & 4.26 & 0.07 & 0.23 & 0.13 \\
& $(0.10)$ & $(1.91)$ & $(0.10)$ & $(0.20)$ & $(0.17)$ \\
\hline $\mathbf{B L}_{\mathrm{S}}$ & 0.23 & 4.46 & 0.12 & 0.27 & 0.13 \\
& $(0.08)$ & $(1.61)$ & $(0.12)$ & $(0.27)$ & $(0.17)$ \\
\hline
\end{tabular}

Table 2. Median and IQR of the analyzed parameters during the different stages of stress session are shown.* reflects significant differences with $\mathrm{BL}_{\mathrm{S}}$, whereas ${ }^{\dagger}$ reflects significant differences with ST and \# reflects significant differences with SA $(p<0.001)$.

\begin{tabular}{lllll} 
& $\mathbf{B L}_{\mathrm{S}}$ & $\mathbf{S T}$ & $\mathbf{S A}$ & $\mathbf{V E}$ \\
\hline $\bar{f}_{\text {res }}(H z)$ & 0.23 & $0.30^{*}$ & $0.28^{*}$ & $0.33^{*, \dagger, \#}$ \\
& $(0.08)$ & $(0.10)$ & $(0.06)$ & $(0.07)$ \\
\hline$\overline{B B}(s)$ & 4.46 & $3.15^{*}$ & $3.73^{*, \dagger}$ & $3.28^{*, \dagger, \#}$ \\
& $(1.61)$ & $(0.62)$ & $(0.95)$ & $(0.73)$ \\
\hline$\alpha_{T_{i n}}$ & 0.12 & $0.02 *$ & $0.02 *$ & $0.01 *, \#$ \\
& $(0.12)$ & $(0.07)$ & $(0.07)$ & $(0.04)$ \\
\hline$\alpha_{T_{e x}}$ & 0.27 & $0.45 *$ & $0.27^{\dagger}$ & $0.35 *, \#$ \\
& $(0.20)$ & $(0.35)$ & $(0.31)$ & $(0.38)$ \\
\hline$\theta($ rad $)$ & 0.13 & $0.37 *$ & $0.20 *, \dagger$ & $0.34 *, \#$ \\
& $(0.17)$ & $(0.33)$ & $(0.28)$ & $(0.34)$ \\
\hline
\end{tabular}

Table 3. Spearman correlation of the different proposed parameters with $\overline{B B}(H z)$ in each stage of the stress session.

\begin{tabular}{lllll} 
& BL $_{\mathrm{S}}$ & $\mathbf{S T}$ & $\mathbf{S A}$ & $\mathbf{V E}$ \\
\hline$\alpha_{T_{\text {in }}}$ & 0.34 & -0.07 & 0.22 & -0.16 \\
\hline$\alpha_{T_{\text {ex }}}$ & 0.07 & 0.01 & -0.08 & -0.05 \\
\hline$\theta($ rad $)$ & -0.04 & 0.00 & -0.16 & -0.03 \\
\hline
\end{tabular}

values of these three variables under stress would reflect a change in the adaptability of the respiratory system, which could be caused by a change in the driving mechanisms of respiration, resulting in an increase of this adaptability during stress. This hypothesis is supported by the absence of correlation of the proposed variables with $\overline{B B}$ (Table 3 ), thus suggesting that some other changes than an increase in respiratory rate do take place during stress.

Despite the fact that further research would be needed in order to identify the stress-related changes in respiratory dynamics reported here with their physiological origin, analysis of these dynamics could be helpful for noninvasive stress assessment.

\section{Conclusions}

Apart from respiratory frequency, respiratory dynamics might also be modified as a response to stress. In this work, three different parameters to characterize those dynamics have been proposed. All of them were significantly modified under stress when compared to a basal state, thus suggesting differences in the underlying mechanisms controlling respiration. These indexes could result helpful in non-invasive stress monitoring.

\section{Acknowledgements}

This work was supported by grant BES-2015-073694 and project TIN2014-53567-R from Ministerio de Economía y Competitividad. Also by Government of Aragón and European Social Fund (EU) through BSICoS group (T96) and by CIBER in Bioengineering, Biomaterials \& Nanomedicine (CIBER-BBN) through Instituto de Salud Carlos III.

\section{References}

[1] Taelman J, Vandeput S, Vlemincx E, Spaepen A, Van Huffel $\mathrm{S}$. Instantaneous changes in heart rate regulation due to mental load in simulated office work. European Journal of Applied Physiology 2011;111(7):1497-1505.

[2] Vrijkotte TG, Van Doornen LJ, De Geus EJ. Effects of work stress on ambulatory blood pressure, heart rate, and heart rate variability. Hypertension 2000;35(4):880-886.

[3] Hernando A, Lázaro J, Gil E, Arza A, Garzón JM, LópezAntón R, de la Cámara C, Laguna P, Aguiló J, Bailón R. Inclusion of respiratory frequency information in heart rate variability analysis for stress assessment. IEEE journal of biomedical and health informatics 2016;20(4):1016-1025.

[4] Dimsdale JE. Psychological stress and cardiovascular disease. Journal of the American College of Cardiology 2008; 51(13):1237-1246.

[5] Bao AM, Meynen G, Swaab D. The stress system in depression and neurodegeneration: focus on the human hypothalamus. Brain research reviews 2008;57(2):531-553.

[6] Aguiló J, Ferrer-Salvans P, García-Rozo A, Armario A, Corbí Á, Cambra FJ, Bailón R, González-Marcos A, Caja G, Aguiló S, et al. Project es3: Attempting to quantify and measure the level of stress. Revista de neurologia 2015; 61(9):405-415.

[7] Peng CK, Mietus JE, Liu Y, Lee C, Hausdorff JM, Stanley HE, Goldberger AL, Lipsitz LA. Quantifying fractal dynamics of human respiration: age and gender effects. Annals of biomedical engineering 2002;30(5):683-692.

Address for correspondence:

Javier Milagro

Dep. Ingeniería Electrónica y Comunicaciones. Universidad de Zaragoza, C/ María de Luna 1, L.3.07, 50018 Zaragoza, Spain milagro@unizar.es 